\title{
Consulta preanalgésica del parto. Un proyecto hecho realidad
}

\author{
V. Aceña Fabian, H. Vasquez Lobo, J.L. Ortega García y F. Neira Reina \\ Servicio de Anestesia, Reanimación y Tratamiento del Dolor. Unidad de Gestión Bloque Quirúrgico. \\ Hospital Universitario Puerto Real. Cádiz
}

Aceña Fabian V, Vasquez Lobo H, Ortega García JL, Neira Reina F. Consulta preanalgésica del parto. Un proyecto hecho realidad. Rev Soc Esp Dolor 2014; 21(6): 315-322.

\begin{abstract}
Objective: The objective of this study is to report the strategy followed in the Hospital Universitario Puerto Real (Cádiz, Spain) to create a pre-analgesic consultation for labor in order to provide an appropriate analgesia during delivery, complying with Law 41/2002 which regulates the autonomy rights and obligations of patients regarding information and clinical documentation. We evaluate the approach taken in the rest of the hospitals in Andalusia.

Material and method: We analyzed the challenges and difficulties that arose at the time and the strategy for the creation of this consultation. The questions which were raised were: At what stage of pregnancy pregnant women should attend the consultation? What staffing and training would be required to create this consultation? Where would it be located? What materials would be needed to properly inform our pregnant women? We conducted a phone survey in order to determine how our response was given to labor analgesia and how Law 41/2002 was being applying in the rest of hospitals in Andalusia.

Results: The pre-analgesic labor consultation of the Hospital Universitario Puerto Real was created in February 2007. Training courses were developed for delivery room staffing. Consultations are currently carried out during the $20^{\text {th }}$ week of gestation, coincident with the echography done by the Gynecology Service of the Hospital. The staffs assigned are a nurse assistant and the anesthesiologist on duty in the delivery room The consultation room is located in the in the outpatient depart-
\end{abstract}

Recibido: 01-08-13.

Aceptado: 01-12-13. ment of the hospital and has a capacity for 12 people. Pregnant women receive a lecture and an informative video. After this, we complete the individualized pre-analgesic assessment of each patient and the informed consent process. Since its creation, they have been cited 12,990 pregnant women. The annual average of pregnant women informed and preoperatively evaluated is of 1,384 ; which represents the $63.93 \%$ of all pregnant women. Twenty-seven of the 33 hospitals of the Andalusia Health System in which deliveries are practiced, telephone survey respondents. The results of this survey show that the pre-anesthetic assessment for pregnant women is only performed in 8 centers $(29.63 \%)$, in 5 of them routinely $(8.52 \%)$ and only to high-risk pregnant women in 3 (11.1\%). The informed consents are obtained before the delivery only in 9 of the 27 hospitals surveyed (33\%). In the 18 centers remaining they were obtained at delivery (66.67\%).

Conclusions: Since the foundation of the pre-analgesic consultation for delivery in our hospital, we have improved the pre-analgesic assessment of pregnant women and we have achieved complying with Law 41/2012.

Key words: Pre-analgesic consultation. Management. Delivery pain. Epidemiology.

\section{RESUMEN}

Objetivo: el objetivo del presente estudio es dar a conocer la estrategia seguida en el Hospital Universitario Puerto Real para crear una consulta preanalgésica del parto con el fin de dar respuesta a los derechos de la embarazada a recibir una adecuada analgesia durante el trabajo del parto, cumpliendo la Ley 41/2002 Reguladora de la autonomía del paciente y de derechos y obligaciones en materia de información y documentación clínica. Valorar la situación en el resto de los hospitales andaluces.

Material y método: analizamos los retos y dificultades que se plantearon en su momento y la estrategia para la creación de esta consulta. Las cuestiones que se plantearon fueron: ¿en qué 
momento de la gestación deberían acudir las gestantes? ¿Qué necesidades de personal y formación de personal supondría la creación de esta consulta? ¿Dónde se ubicaría la consulta? ¿Qué material didáctico sería necesario para informar adecuadamente a nuestras gestantes? Para determinar cómo se había dado respuesta a la analgesia del parto y la aplicación de la Ley 41/2002 en el resto de los hospitales andaluces realizamos una encuesta telefónica.

Resultados: la consulta de preanalgesia para el parto comenzó en nuestro hospital en febrero del año 2007. Se desarrollaron cursos de formación para el personal de paritorio. La consulta se realiza en la semana 20 de gestación haciéndola coincidir con la ecografía que realiza el Servicio de Ginecología en la semana 20. El personal asignado es una auxiliar de enfermería y el anestesiólogo de guardia asignado a paritorio. La consulta se ubica en una sala en el Hospital de Día con capacidad suficiente para 12 personas, donde se imparte una charla y se proyecta un vídeo informativo. Posteriormente se hace una valoración individualizada, se cumplimenta y firma el consentimiento informado. Desde la creación de la consulta de preanalgesia del parto han sido citadas en la consulta 12.990 gestantes. La media anual de gestantes informadas y valoradas preoperatoriamente es de 1.384 , lo que supone un $63,93 \%$ del total de las gestantes. Los datos obtenidos de las encuestas telefónicas realizadas en los Centros Hospitalarios del Sistema Andaluz de Salud, corresponden a 27 de los 33 centros hospitalarios en los que se atienden partos. La valoración preanestésica a las gestantes sólo se realiza en 8 centros (29,63\%), en 5 de ellos de forma rutinaria (18,52 \%) y en $3(11,1 \%)$ sólo a las gestantes de alto riesgo. El consentimiento informado se obtiene previamente al momento del parto tan sólo en 9 de los 27 hospitales encuestados (33,33\%), en los 18 centros restantes se obtiene en el momento del parto $(66,67 \%)$.

Conclusiones: desde que se instauró la consulta de preanalgesia del parto en nuestro hospital se ha mejorado la valoración preanalgésica de la gestante y hemos conseguido dar cumplimiento a la Ley 41/2012.

Palabras clave: Consulta preanalgesia. Gestión. Dolor del parto. Epidemiología.

\section{INTRODUCCIÓN}

La gran mayoría de las mujeres sufren dolor durante la progresión de su trabajo del parto. El modo de experimentar este dolor depende de circunstancias culturales y sociales, así como de aspectos individuales de tipo cognitivo, emocional y motivacional (1). El profesor Bonica indicaba que este dolor podía llegar a ser tan intenso que resultase intolerable. Melzack demostró, en 1984, comparando el dolor de parto con otros dolores, que este es uno de los más severos que puede presentarse en el ser humano (2). Muchas mujeres, especialmente las nulíparas, califican el dolor del parto como muy severo o intolerable $(3,4)$.

Está demostrado que el control del dolor del parto se asocia a una mayor satisfacción materna (5). Las mujeres deben participar en la toma de decisiones sobre aspectos del parto, incluyendo el alivio del dolor, antes del inicio del trabajo del parto. Esto se puede lograr mediante la educación e información durante el embarazo, de las diferentes técnicas de alivio del dolor de parto. Esto facilitaría la valoración de las diferentes opciones y la toma de decisiones, por parte de la gestante, antes de iniciarse el parto, evitando la angustia física y el estrés emocional del dolor durante el trabajo del parto.

El dolor tiene como objetivo alertar a la gestante del comienzo del parto. Una vez iniciado el parto el dolor carece de utilidad y, sin embargo, sí tiene repercusiones adversas sobre la madre, que secundariamente afectan al feto (6).

Toda técnica analgésica aplicada a la embarazada debe ser segura, eficaz y viable. Tiene que tener una mínima incidencia de efectos secundarios, la menor interferencia posible con la dinámica del parto, un alto porcentaje de éxitos en alivio del dolor, no tener contraindicaciones maternas ni fetales y disponer de la organización y los recursos necesarios.

En relación a la seguridad materna, las técnicas de bloqueo regional parecen ser, indiscutiblemente, las más seguras. En cuanto a la eficacia y la calidad analgésica, la técnica epidural es muy superior a las demás. Donde existe una gran controversia es en la interferencia de la analgesia epidural sobre la progresión del parto (7).

Los métodos utilizados en el tratamiento del dolor del parto pueden ser farmacológicos y no farmacológicos. Los no farmacológicos consisten en terapias psicológicas para el control de la ansiedad y el dolor del parto. Entre los métodos farmacológicos se pueden utilizar la analgesia inhalatoria con $\mathrm{NO}_{2}$, la analgesia sistémica con opioides, fundamentalmente con meperidina, las técnicas locorregionales, neuroaxiales y los bloqueos nerviosos no espinales (8).

La analgesia epidural proporciona un alivio del dolor superior a la analgesia no epidural en el trabajo de parto. La analgesia epidural se asocia a una prolongación del segundo estadio del parto e incrementa el riesgo de parto instrumentado, sin aumentar la incidencia de cesárea, con un nivel de evidencia I $(9,10)$.

En Estados Unidos, las técnicas neuroaxiales se utilizan en más del $70 \%$ de las parturientas en los hospitales con más de 1.500 partos al año (11). En los hospitales andaluces se ha producido un aumento progresivo de la analgesia epidural, que ha pasado del $15 \%$ en el 2000 al $50 \%$ en 2005. En los siguientes cinco años, siguió el incremento del número de partos con analgesia epidural, llegando en el año 2010 a valores próximos al $60 \%$ (12).

En términos generales, las gestantes debe elegir de forma libre y voluntaria el tratamiento para el dolor de trabajo del parto. Durante el embarazo se debe informar a las gestantes acerca de los beneficios y los efectos adversos potenciales, de los diferentes métodos de control del dolor (8).

Aunque las gestantes suelen ser mujeres sanas y jóvenes, y el parto no es una situación patológica, siempre debe 
hacerse una valoración y examen físico antes de cualquier procedimiento anestésico.

Los objetivos de la visita preanalgésica en obstetricia son: establecer un contacto personal con la gestante antes del parto, informarle de todo lo referente a la analgesia epidural, reducir la ansiedad, identificar los factores de riesgo, patologías y tratamientos que interfieran con la analgesia y obtener el consentimiento informado.

La analgesia epidural no se debe improvisar, la gestante debe meditar con calma si realmente quiere someterse a este procedimiento. Por tanto, es muy importante que un anestesiólogo le informe del proceso, de las complicaciones y riesgos de la técnica. Además, debe realizarse una adecuada valoración física y pruebas complementarias para determinar si la gestante es apta o no para esta técnica.

Por otro lado es imprescindible obtener el consentimiento informado previo. Lo ideal es obtenerlo con antelación, antes de iniciarse el trabajo del parto. En el momento del parto las gestantes no suelen estar en condiciones de recibir información, valorarla y tomar una decisión sobre esta técnica invasiva.

Dentro del Proceso Asistencial Integrado del Embarazo, Parto y Puerperio del Servicio Andaluz de Salud, se establece que aproximadamente a las 36 semanas de embarazo, en la $8^{\text {a }}$ visita de la gestante a su médico de atención primaria y/o su matrona de zona, recibe información de analgesia en el parto. Se debe fomentar la participación y el asesoramiento del anestesiólogo. Además se le entrega un folleto informativo sobre la analgesia en el parto (Documento de información clínica específica y el Documento de Declaración de consentimiento). En este proceso asistencial se establece como indicador de calidad la oferta de técnicas de analgesia, incluyendo la epidural, a todas las gestantes (13).

El objetivo del presente estudio es dar a conocer la estrategia seguida en el Hospital Universitario Puerto Real para crear una consulta preanalgésica del parto, con el fin de dar respuesta a los derechos de la embarazada a recibir una adecuada analgesia durante el trabajo del parto, cumpliendo la Ley 41/2002 Reguladora de la autonomía del paciente y de derechos y obligaciones en materia de información y documentación clínica. Valorar la situación en el resto de los hospitales andaluces.

\section{MATERIAL Y MÉTODOS}

Se realizó un estudio observacional descriptivo. En primer lugar obtuvimos datos procedentes de la Unidad de Documentación Clínica de nuestro Hospital referentes al número de gestantes atendidas, número de partos, número de cesáreas, porcentaje de epidurales para parto y cesárea desde el año 2007 al 2012. Además obtuvimos el número de gestantes a las que se les había realizado una valoración preanalgésica obstétrica durante este periodo.

Teniendo en cuenta el elevado porcentaje de mujeres que solicitaban y recibían analgesia epidural para el parto en el año 2007 y la necesidad de aplicar la Ley 41/2002 de autonomía del paciente, se planteó la posibilidad de crear una consulta de preanalgesia del parto.

Analizamos los retos y dificultades que se plantearon en su momento y la estrategia para la creación de esta consulta. El objetivo de la consulta era informar a la gestante de las posibilidades de analgesia para el parto, centrándose en la analgesia epidural, además de realizar una adecuada valoración preoperatoria y obtener el consentimiento informado con antelación al momento del parto. Las cuestiones que se plantearon fueron las siguientes: ¿en qué momento de la gestación deberían acudir las gestantes? ¿Qué necesidades de personal y formación de personal supondría la creación de esta consulta? ¿Dónde se ubicaría la consulta? ¿Qué material didáctico sería necesario para informar adecuadamente a nuestras gestantes?

Para determinar cómo se había dado respuesta a la analgesia del parto y la aplicación de la Ley 41/2002 en el resto de los hospitales andaluces realizamos una encuesta telefónica. El objetivo era conocer si el resto de los hospitales del Servicio Andaluz de Salud tenían una consulta de analgesia epidural para el parto o, en su defecto, realizaban algún tipo de charla informativa y cómo realizaban la valoración preanalgésica obstétrica. Para ello, efectuamos una encuesta a través de un cuestionario (Tabla I) que se completó telefónicamente, contactando durante los meses de abril y mayo de 2013 con los Servicios de Anestesia y Reanimación de los diferentes hospitales andaluces.

\section{RESULTADOS}

La consulta de analgesia epidural para el parto comenzó en nuestro hospital en febrero del año 2007. Se desarrollaron cursos de formación para las matronas y el personal de nuestro centro implicado en el proceso. La consulta se realiza en la semana 20 de gestación haciéndola coincidir con la ecografía que realiza el Servicio de Ginecología en la semana 20 (ECO 20), evitando a la gestante desplazamientos adicionales al hospital. El personal asignado es una auxiliar de enfermería y el anestesiólogo de guardia asignado a paritorio. La consulta se ubica en una sala en el Hospital de Día con capacidad suficiente para 12 personas.

Se creó un cuestionario (Fig. 1) que se reparte a las gestantes, junto con el consentimiento informado, para optimizar el tiempo en el momento de la valoración individualizada de cada una de ellas.

Se imparte una charla y se proyecta un vídeo informativo sobre la analgesia del parto, filmado en el hospital. La consulta se desarrolla en dos sesiones a lo largo de la mañana para poder compatibilizarla con la consulta de ECO 20. 
Servicio Andaluz de Salud

CONSEJERÍA DE SALUD Y BIENESTAR SOCIAL

IUMTA DE AMDALUCIP SERVICIO DE ANESTESIOLOGÍA Y REANIMACIÓN HOSPITAL UNIVERSITARIO PUERTO REAL (CÁDIZ)

CONSULTA PREANESTÉSICA PARA ANALGESIA EPIDURAL EN EL PARTO

Nombre y apellidos:

Edad:

$\mathrm{N}^{\mathrm{o}}$ de historia:

Espacio para etiqueta identificativa

$\mathrm{N}^{\circ} \mathrm{SS}$ :

Fecha:

\section{Semana de gestación:}

\section{Antecedentes personales:}

¿Padece o ha padecido alguna enfermedad de relevancia?

En caso afirmativo, ¿cuál?:

¿Ha sido anestesiado en alguna ocasión?

En caso afirmativo, ¿qué tipo de anestesia le aplicaron?:

GENERAL RAQUÍDEA

¿Tuvo alguna incidencia durante o después de la anestesia? En caso afirmativo, ¿cuáles?:

¿Es alérgica a algún medicamento?

En caso afirmativo, ¿a cuál?:

¿Toma alguna medicación?

En caso afirmativo, ¿cuál?:

\section{Exploración física:}

Talla (cm):

Otros datos de interés:

$$
\text { Peso (kg): }
$$

\section{Analítica:}

Hemograma: NORMAL

ANORMAL: $\mathrm{Hb}$

HCT

EPIDURAL

SÍ

NO

Sí

NO

Coagulación: NORMAL ANORMAL:TP TPTA
Firma y DNI de la paciente

Firma médico

HOSPITAL UNIVERSITARIO PUERTO REAL

Carretera Nacional IV - km 665, 11510 PUERTO REAL (CÁDIZ)

Tel. 956005000

Fig. 1. 


\section{TABLA I. CUESTIONARIO TELEFÓNICO}

1. ¿Se realiza en su centro algún tipo de charla informativa sobre la técnica epidural para la analgesia del parto?

2. En caso de organizarse estas charlas, ¿quién o quiénes las imparten?

3. ¿Se realizan en su centro valoraciones preanestésicas a las gestantes que van a someterse a la técnica de anestesia epidural?

4. ¿En qué momento se firma el consentimiento informado para la técnica epidural?

Se explican las indicaciones, riesgos, efectos secundarios y contraindicaciones, así como las alternativas disponibles a la analgesia epidural y se anima a las gestantes a que expongan sus dudas y temores. Posteriormente se hace una valoración individualizada, recogiendo sus antecedentes personales y efectuando una exploración física, se les entrega un formulario informativo, se cumplimenta y firma el consentimiento informado escrito para "Analgesia en el parto: técnica epidural" del Servicio Andaluz de Salud. Las gestantes se llevan una copia de este consentimiento y en cualquier momento pueden revocarlo.

Cuando las gestantes acuden al hospital para el proceso del parto, deben traer este consentimiento, junto con una analítica que se realiza en el último trimestre del embarazo, en su centro de salud. De este modo, el anestesiólogo de guardia valorará conjuntamente la historia clínica y las pruebas complementarias necesarias previamente a la realización de la técnica analgésica más adecuada.

En base a los datos obtenidos de la Unidad de Documentación Clínica del Hospital Universitario Puerto Real, del año 2007 al 2012, se atendieron una media anual de 2.165 gestantes, de las cuales 1.525 finalizaron la gestación por parto vaginal y 529 por cesárea. La media del porcentaje de analgesia epidural durante este periodo fue del 78,32\%. En la tabla II se muestran los datos desglosados por años.

Desde la creación de la consulta de preanalgesia epidural para el parto han sido citadas en la consulta 12.990 gestantes, de las cuales acudieron 8.307. La media anual de gestantes informadas y valoradas preoperatoriamente fue de 1.384, lo que supone un $63,93 \%$ de las gestantes. Un $81,71 \%$ de las analgesias epidurales administradas para el parto habían pasado por nuestra consulta, quedando un $18,22 \%$ que fueron informadas y valoradas en el momento del parto, antes de la administración de la analgesia epidural. En la tabla III se muestran los datos desglosados por años.

Los datos obtenidos de las encuestas telefónicas realizadas en los centros hospitalarios del Sistema Andaluz de Salud corresponden a 27 de los 33 centros hospitalarios en los que se atienden partos. De estos centros, sólo se organizan charlas informativas en 8 de ellos $(29,63 \%)$, en los 19 centros restantes $(70,37 \%)$ no se organiza ningún tipo de charla informativa a las gestantes. De los 8 centros en los que se imparten las charlas tan sólo en 2 son impartidas por anestesiólogos, mientras que en el resto de los centros son impartidas por una enfermera con la especialidad de matrona. En lo referente a la valoración preanestésica a las gestantes sólo se realiza en 8 centros $(29,63 \%)$, en 5 de ellos de forma rutinaria $(18,52 \%)$ y en $3(11,1 \%)$ sólo a las gestantes de alto riesgo. El consentimiento informado se obtiene previamente al momento del parto tan sólo en 9 de los 27 hospitales encuestados $(33,33 \%)$, en los 18 centros restantes se obtiene en el momento del parto $(66,67 \%)$. Toda la información obtenida de las encuestas telefónicas esta detallada en las figuras 2,3 y 4 .

\section{TABLA II.}

\begin{tabular}{|c|c|c|c|c|c|c|c|}
\hline & Añ̃o 2007 & Añ̄o 2008 & Año 2009 & Año 2010 & Año 2011 & Año 2012 & Media \\
\hline Número de gestantes & 2.295 & 2.250 & 2.067 & 2.139 & 2.162 & 2.077 & $2.165,00$ \\
\hline Número de partos & 1.799 & 1.733 & 1.485 & 1.585 & 1.659 & 1.554 & $1.635,83$ \\
\hline Número de cesáreas & 496 & 517 & 582 & 554 & 503 & 523 & 529,17 \\
\hline Epidurales en partos & 1.225 & 1.254 & 1.187 & 1.176 & 1.284 & 1.043 & $1.194,83$ \\
\hline Epidurales en cesáreas & 467 & 488 & 520 & 535 & 486 & 501 & 499,50 \\
\hline$\%$ Partos con epidural & $68,09 \%$ & $72,36 \%$ & $79,93 \%$ & $74,20 \%$ & $77,40 \%$ & $67,12 \%$ & $73,18 \%$ \\
\hline \% Cesáreas con epidural & $94,15 \%$ & $94,39 \%$ & $89,35 \%$ & $96,57 \%$ & $96,62 \%$ & $95,79 \%$ & $94,48 \%$ \\
\hline Total epidurales & 1.692 & 1.742 & 1.707 & 1.711 & 1.770 & 1.544 & $1.694,33$ \\
\hline$\%$ Epidurales & $73,73 \%$ & $77,42 \%$ & $82,58 \%$ & $79,99 \%$ & $81,87 \%$ & $74,34 \%$ & $78,32 \%$ \\
\hline
\end{tabular}


TABLA III.

\begin{tabular}{|c|c|c|c|c|c|c|c|}
\hline & 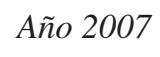 & Año 2008 & 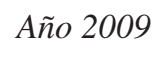 & Año 2010 & Aก̃ 2011 & Año 2012 & Media \\
\hline Citados en consulta epidural & 2.295 & 2.250 & 2.067 & 2.139 & 2.162 & 2.077 & $2.165,0$ \\
\hline Presentados en consulta epidural & 1.382 & 1.489 & 1.277 & 1.619 & 1.263 & 1.277 & $1.384,5$ \\
\hline$\%$ Asistencia citados & $60,22 \%$ & $66,18 \%$ & $61,78 \%$ & $75,69 \%$ & $58,42 \%$ & $61,48 \%$ & $63,96 \%$ \\
\hline$\%$ Epidurales con consulta previa & $81,68 \%$ & $85,48 \%$ & $74,81 \%$ & $94,62 \%$ & $71,36 \%$ & $82,71 \%$ & $81,78 \%$ \\
\hline$\%$ Valoraciones epidural in situ & $18,32 \%$ & $14,52 \%$ & $25,19 \%$ & $5,38 \%$ & $28,64 \%$ & $17,29 \%$ & $18,22 \%$ \\
\hline \multicolumn{8}{|c|}{ Total de gestantes citados periodo 2007-2012: 12.990} \\
\hline Total de gestantes que efectuaron & onsulta epic & lural en este & periodo: 8.3 & & & & \\
\hline
\end{tabular}

\section{DISCUSIÓN}

En nuestro hospital se oferta analgesia epidural para el parto las 24 horas al día, los 365 días del año.

Hasta febrero de 2007 el Servicio de Anestesiología y Reanimación sólo seguía las directrices del Proceso Asis-

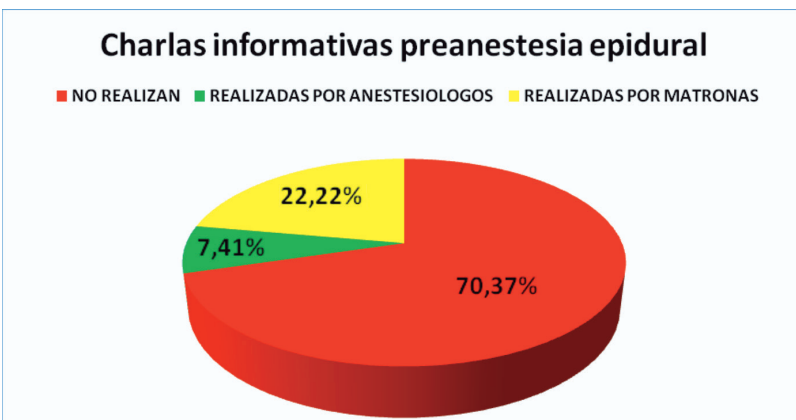

Fig. 2.

\section{Valoracion preanestésica previa}

—RUTINARIAMENTE $\because$ SOLO PACIENTES DE RIESGO $\amalg$ NOREALIZAN

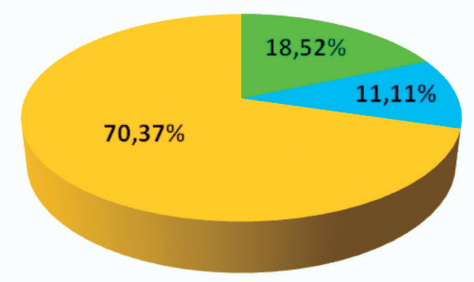

Fig. 3. tencial Integrado del Embarazo, Parto y Puerperio del Servicio Andaluz de Salud (13). De este modo, el primer contacto con la gestante ocurría en el momento del parto en el que se evaluaba a la gestante y se comprobaba el consentimiento informado recibido en atención primaria. En el caso de no tener este consentimiento se informaba a la paciente para que firmara este consentimiento.

Según la Ley 41/2002 de autonomía del paciente, todos los pacientes tienen derecho a ser informados ante cualquier actuación en el ámbito de su salud. Los profesionales que apliquen una técnica o procedimiento durante el proceso asistencial serán los responsables de ofrecer esta información. Los pacientes, una vez recibida esta información, deben dar su conformidad de forma libre y voluntaria, siendo conscientes de los riesgos (14).

En el momento del parto, las gestantes están sometidas a una situación dolorosa, muchas veces estresante, que no les permite asimilar la información que se les da y tomar una decisión de forma libre y voluntaria. En la mayoría de las ocasiones lo único que desean es que se les alivie el dolor sin considerar los riesgos que conlleva la técnica.

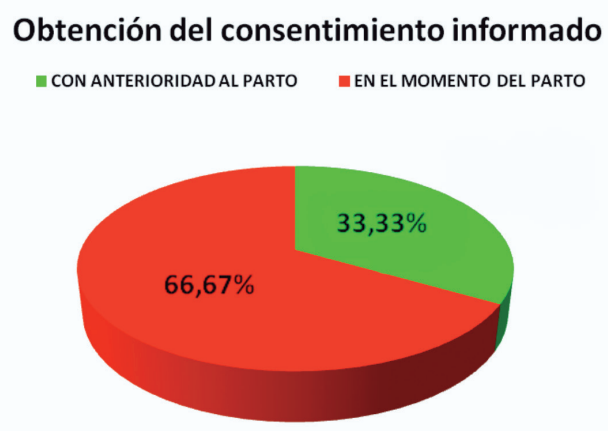

Fig. 4. 
Lo aconsejable sería una visita preanestésica con antelación suficiente, como la que se lleva a cabo en cualquier cirugía, pero esta no resulta fácil ya que en muchos centros hospitalarios lo habitual es que la gestante acuda al centro sólo para el parto. La gestante con patología asociada o con patología obstétrica siempre debe acudir a una visita preanestésica $(15,16)$.

Con la consulta de preanalgesia para el parto, conseguimos cumplir la Ley 41/2002, obteniendo un consentimiento informado en condiciones óptimas. Además, nos permite identificar los factores de riesgo, patologías y tratamientos que interfieren con la analgesia y reducimos la ansiedad de las gestantes, que de este modo, tienen su primer contacto con el anestesiólogo.

La actividad de la consulta está coordinada entre el Servicio de Anestesia y Reanimación y el Servicio de Ginecología y Obstetricia. Con el objetivo de que las gestantes sólo acudan un día al hospital, se citan coincidiendo con el día de la consulta para la ecografía de las 20 semanas. Esta consulta queda registrada en Diraya (Sistema de información del Sistema Sanitario Andaluz).

Aunque muchas de las gestantes son valoradas en nuestra consulta, en algunas ocasiones llegan al hospital gestantes que no acudieron a la misma por falta de coordinación, embarazadas itinerantes o mala información con las matronas de atención primaria. Estas son valoradas en el momento del parto, son informadas y se les solicita que firmen el consentimiento informado si están conformes. De este modo, se garantiza la calidad asistencial en el parto, ofertando en todo momento la analgesia epidural.

Cada hospital debe disponer de un protocolo para la analgesia del parto y, dentro de este, sería deseable la existencia de una consulta de evaluación preanalgésica de la paciente obstétrica.

Con nuestro estudio hemos evidenciado que son muy pocos los centros hospitalarios que organizan charlas informativas a las gestantes y que, en un muy pocos casos, son dirigidas por anestesiólogos. Probablemente, los centros que no las organizan se limitarán a seguir las directrices del Proceso Asistencial Integrado del Embarazo, Parto y Puerperio del Sistema Andaluz de Salud, dejando en manos de las matronas de atención primaria la labor de informar sobre las técnicas de analgesia para el parto. Sería conveniente que se fomentara la participación activa de los anestesiólogos de los centros hospitalarios.

Por otro lado, hemos detectado que en general no se realiza de forma rutinaria una valoración a la gestante con antelación al momento del parto. La valoración que hacemos en nuestra consulta nos permite detectar todos aquellos factores que pueden influir en la técnica epidural, con anticipación, facilitando enormemente nuestra labor asistencial en el momento de parto.

Por último, los resultados de nuestra encuesta muestran que, en la mayoría de los centros, se obtiene el consenti- miento informado de analgesia del parto en el momento del mismo (17). En ese momento, la gestante está altamente condicionada por el dolor del trabajo del parto y, en cierto modo, no toma una decisión de acuerdo con su propia y libre voluntad, valorando adecuadamente los riesgos existentes.

Desde que se instauró la consulta de preanalgesia del parto en nuestro hospital se ha mejorado la valoración preanalgésica de la gestante y hemos conseguido dar cumplimiento a la Ley 41/2012 (14), obteniendo con antelación suficiente el consentimiento informado. Somos conscientes de que, en muchas ocasiones, la falta de coordinación con atención primaria impide que todas las gestantes sean informadas y valoradas en el hospital.

El Servicio de Anestesia del Hospital Universitario Puerto Real sigue dando difusión a esta consulta para mejorar la atención sanitaria, intentado proporcionar la mejor calidad asistencial posible a nuestras pacientes.

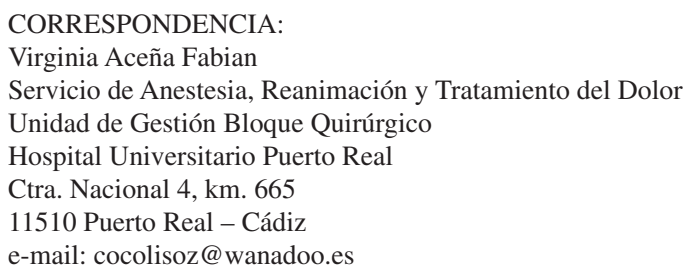

\section{BIBLIOGRAFÍA}

1. Lowe NK. The Nature of labor pain. Am J Obstet Gynecol 2002; 186:16-24.

2. Melzack R. The myth of painless childbirth. The John J. Bonica Lecture Pain. 1984;19:321-37.

3. Ranta P, Spalding M, Kangas-Saarela T, Jokela R, Hollmén A, Jouppila P, et al. Maternal expectations and experiences of labor pain-options of 1091 finnish parturients. Acta Anaesthesiol Scand 1995; 39:60-6.

4. Melzak R, Taenzer P, Feldman P, Kinch RA. Labour is still painful after prepared childbirth training. Can Med Assoc J 1981;125:357-63.

5. McCrea BH, Wright ME. Satisfaction in childbirth and perceptions of personal control in pain relief during labor. J Adv Nurs 1999;29:877-84.

6. Brownridge P. The nature and consequences of childbirth pain. Eur J Obstet Gynecol Reprod Biol 1995;59(Supl.):915.

7. López Monserrat JC. Analgesia epidural para trabajo del parto. Ob Stare: el mundo de la maternidad. Primavera 2004;12:23-7.

8. Jones L, Othman M, Dowswell T, Alfirevic Z, Gates S, Newburn M, et al. Pain management for women in labour: An overiew of systematyc reviews. Cochrane Database Syst Rev 2012;3:CD009234.

9. Australiand and New Zealand College of Anaesthetists and Faculty of Pain Medicine. Acute pain Management: Scientific Evidence. Third Edition 2010:390-4. 
10. ASA Task Force on Obstetric Anesthesia. 2006. Disponible en: http//www.Asahq.org/publications.ASA

11. Bucklin BA, Hawkins JL, Anderson JR, Ullrich FA. Obstetric anesthesia workforce survey: Twenty-year update. Anesthesiology 2005;103:645-53.

12. Escuela Andaluza de Salud Pública. Servicio Andaluz de Salud. Consejería de Salud. Junta de Andalucía. Resultados y Calidad del Sistema sanitario de Andalucía: Edición 2012. Disponible en: http://www.calidadsaludandalucia.es/ es/documento.html

13. Consejería de salud. Junta de Andalucía. Embarazo, Parto y Puerperio: Proceso Asistencial Integrado. $2^{\mathrm{a}}$ ed. 2005. Disponible en: http://www.juntadeandalucia.es/servicios/ publicaciones/detalle/39719.html
14. Ley $41 / 2002$, de 14 de noviembre, básica reguladora de la autonomía del paciente y de derechos y obligaciones en materia de información y documentación clínica. BOE $\mathrm{n}^{\circ}$ 274 del 15 de noviembre de 2002.

15. López Gil MV, Manrique Muñoz S. Anestesia obstétrica en casos de cirugía. En: Cabero Roura L, Rodríguez Saldívar D, editores. Operatoria obstétrica. Una visión actual. México: Médica Panamericana; 2009. p. 357.

16. Hinova A. Fernando R. The preoperative assesment of obstetric patients. Best Pract Res Clin Obstet Gynaecol 2010;24(3):261-76.

17. Orden de 8 de julio de 2009 por la que se dictan instrucciones a los Centros del sistema Sanitario Público de Andalucía, en relación al procedimiento de Consentimiento Informado. BOJA n ${ }^{\circ} 152$ del 6 de agosto de 2009. 